\title{
Mass Variation Rate, in Acidic Environment, of Polyhydroxybutyrate and Chitosan matrices with Potential Application as Controlled Drug Delivery System
}

\author{
Talita Goulart da Silva ${ }^{a}$, Vinícius Guedes Gobbi ${ }^{a}$, Bruna Nunes Teixeira ${ }^{b}{ }^{\circledR}$, Tiago dos Santos Mendonçac,
}

Thassio Brandão Cubiça ${ }^{a}$, Layla Ferraz Aquino ${ }^{a}$, Jean Araújo das Neves Silva ${ }^{b}$, Rossana Mara da Silva

\author{
Moreira Thiréb ${ }^{b}$, Roberta Helena Mendonça ${ }^{a *}$ \\ ${ }^{a}$ Departamento de Engenharia Química, Universidade Federal Rural do Rio de Janeiro - UFRRJ, \\ Seropédica, RJ, Brasil \\ ${ }^{b}$ Programa de Engenharia Metalúrgica e de Materiais - COPPE, Universidade Federal do Rio de \\ Janeiro - UFRJ, Rio de Janeiro, RJ, Brasil \\ 'Departamento de Física Teórica, Instituto de Física Armando Dias Tavares, Universidade do Estado do \\ Rio de Janeiro - UERJ, Rio de Janeiro, RJ, Brasil
}

Received: December 08, 2018; Revised: June 24, 2019; Accepted: June 25, 2019

Polymers such as the biocompatible polyhydroxybutyrate (PHB) and the polysaccharide chitosan (Chi), a pH-sensitive polymer, have been studied for drug delivery applications and scaffolds production. In this work, matrices of $\mathrm{PHB}$ and Chi were produced by compression molding $\left(\mathrm{S}_{\mathrm{PHB}-\text { Chi }}\right)$ and loaded with a broad-spectrum biocide chlorhexidine $(\mathrm{CHX})\left(\mathrm{S}_{\mathrm{PHB}-\mathrm{Chi}-\mathrm{CHX}}\right)$. The matrices were analyzed by scanning electron microscope (SEM). The $\mathrm{S}_{\text {PHB-Chi }}$ capacity to absorb and deliver CHX was evaluated by UV-Vis. The antibacterial activity of the samples against $S$. aureus, $P$. aeruginosa and $E$. coli (most common pathogens that cause infection in several body sites) was evaluated using the disk-agar diffusion method. The CHX release results were fitted by the Power Law model. The results suggested that the morphology and the CHX delivery profile were directly related to the Chi amount in the samples and also showed that the $\mathrm{S}_{\text {PHB-Chi-CHX }}$ is able to inhibit the microorganism proliferation, constituting it as a potential candidate for oral applications.

Keywords: Modelling, S. aureus, P. aeruginosa, E. coli, Chlorhexidine.

\section{Introduction}

Some polymers present a transition in their configuration according to the $\mathrm{pH}$ of the surrounding medium and, for this reason, they are classified as $\mathrm{pH}$-sensitive polymers. The physical properties of the polymer, such as solubility and configuration can be adapted by altering the $\mathrm{pH}$. Many $\mathrm{pH}$-sensitive polymers, such as poly(acrylic acid), alginate and chitosan (Chi), have been used in different delivery systems ${ }^{1}$. The versatility of these polymers has increased the interest for using them in potential biomedical applications, since they enable the delivery of drugs, genes and proteins ${ }^{2}$.

The technology of controlled release of drugs may, potentially, solve many problems related to the traditional administration of active principles by regulating the rate and spatial localization of the release agent ${ }^{3}$. The development of materials with antimicrobial properties for medical purposes requires the application of a component that works as a vehicle to deliver the antimicrobial compound. Topical therapy to treat an injured place has shown several advantages, such as avoiding the systemic effects of treatment ${ }^{4,5,6}$. Biodegradable polymers, such as microbial polyesters, have been investigated for biomedical applications. Among them, the bioresorbable and biocompatible polyhydroxybutyrate (PHB) and the polysaccharide Chi have been extensively studied ${ }^{7,8,9,10,11}$.
These materials can be applied for scaffolds production and for the design of materials for drug delivery.

Due to its good biocompatibility and biodegradability, Chi is versatile when considered the route of administration, being compatible with non-invasive delivery routes. Because of the amino groups present in its structure $\mathrm{Chi}$ is $\mathrm{pH}$ responsive and it can provide a cationic ion. The $\mathrm{pKa}$ of Chi is 6.5 , which limits its water solubility under physiological conditions ${ }^{1}$. Chi acts as a soluble cationic polyelectrolyte in dilute acidic solutions, which facilitates effective encapsulation of drugs or proteins by electrostatic interactions ${ }^{1,12}$.

The mechanical properties and potential application to tissue regeneration of $\mathrm{PHB}$ disposables are improved by the incorporation of Chi. PHB degradation occurs slower than Chi degradation, therefore, $\mathrm{PHB} / \mathrm{Chi}$ scaffolds can be applied to drug delivery systems ${ }^{13,14}$. During the implantation and/ or tissue formation, it is possible to occur the emergence of polymicrobial contamination to the biomaterial surface, damaging the implanted area and the adjacent tissue. Chlorhexidine (CHX) is an antimicrobial agent widely applied in medical applications, because it is a broad-spectrum biocide, effective against gram-positive bacteria, gram-negative bacteria and fungi $1^{15,16,17}$.

Chi is a polymer that attracts attention for applications in mucosal drug delivery as a result of its bioadhesive properties, which increases the retention time on the oral mucosa. 
This interaction between Chi and the oral mucosa, at physiological $\mathrm{pH}$, is mainly due to electrostatic attraction with the negatively charged mucin, in combination with contribution of hydrogen bonding and hydrophobic effects ${ }^{6,18}$. Onnaintyet al. ${ }^{6}$ studied release systems based on Chi and montmorillonite for CHX oral mucosal delivery. They observed that the release profiles of CHX from the carriers suggested the diffusion through a swollen matrix and water filled pores due to the controlled drug release mechanism. Senel et al. ${ }^{19}$ designed a formulation containing Chi for local delivery of $\mathrm{CHX}$ to the oral cavity and gels (at 1 or $2 \%$ concentration) or film forms of Chi were prepared containing 0.1 or $0.2 \% \mathrm{CHX}$. The release of CHX from gels was maintained for $3 \mathrm{~h}$ and a prolonged release was observed with film formulations. Giunchediet $\mathrm{al}^{20}$. evaluated the CHX buccal tablets prepared using drug-loaded Chi microspheres. The in vivo results of this study suggested that such tablets are potential candidates for intraoral drug delivery.

According to Mendonça et $\mathrm{al}^{13}$, 3D scaffolds may be produced based on Chi swelling behavior into PHB matrices. The production of scaffolds with both, bulk and surface, functionalized with Chi is one of the main benefits provided by this technique, since bulk and surface functionalization help in the achievement of specific cell responses. Therefore, this technique may be eventually applied for different biological fluids to produce scaffolds which may behave as drug delivery systems ${ }^{13}$.

There are several kinetic models, which describe the overall mechanism of the drug release according to the physical form, its mode of diffusion and polymer matrix characteristics (porous or dense) ${ }^{21}$. Some empirical models may satisfactorily describe the experimental data based on theoretical considerations, such as the Power Law model.

The aim of this work was to develop matrices of PHB and Chi, a $\mathrm{pH}$-sensitive polymer and evaluate the barrier effect promoted by PHB through the mass variation rate in acidic environment. In addition, the ability to incorporate and release CHX was also evaluated in this study. Focusing on understanding the behavior of the CHX release, the results were fitted by the Power Law model.

\section{Experimental}

\subsection{Materials}

Food-grade Chi with a $22 \%$ degree of acetylation was purchased from Polymar SA (Ceará, Brazil). PHB, in white powder form, with a molecular weight (Mw) of 524.000 $\mathrm{g} \mathrm{mol}^{-1}$ was supplied by PHB Industrial S/A (São Paulo, Brazil). Both polymers were used as received. CHX 0,2\% was purchased from Mil Formulas pharmacy and acetic acid was purchased from VETEC.

\subsection{Matrices preparation}

The process for the production of matrices was carried out in three steps. In the first step, the samples were produced by compression molding using a hydraulic press (SOLAB, model SL-11), adopting the following parameters: charge of 4 ton, pressure time of 15 minutes and temperature equal to $165^{\circ} \mathrm{C}$. Mixtures with different ratios of $\mathrm{PHB}$ and $\mathrm{Chi}$ were compressed and the samples were named according to the weight percentage (wt \%) of Chi: $\mathrm{S}_{\mathrm{PHB}: \mathrm{Chi}} 75 / 25(75 \%$ PHB and 25\% Chi); $\mathrm{S}_{\text {PHB:Chi }} 50 / 50$ (50\% PHB and 50\% Chi); $\mathrm{S}_{\mathrm{PHB}: \text { Chi }} 40 / 60(40 \% \mathrm{PHB}$ and $60 \% \mathrm{Chi})$. In the second step, the compressed samples were immersed in $\mathrm{CHX}$ solution $\left(2 \mathrm{mg} \cdot \mathrm{mL}^{-1}\right)$ for $5,15,30,45$ and 60 minutes to evaluate the time effect in $\mathrm{CHX}$ incorporation. In the third step, the samples immersed in $\mathrm{CHX}$ solution for $60 \mathrm{~min}$ were frozen at $-4^{\circ} \mathrm{C}$ and then, lyophilized.

\subsection{Study of the mass variation of the samples in acidic environment}

The mass variation of the matrices was evaluated in acetic acid solution $(1 \% \mathrm{v} / \mathrm{v})$ and the experiments were performed in triplicate. The mass variation was determined using Equation 1. This equation may also be applied to evaluate the matrices swelling degree. However, in this work it will be used the term "mass variation" because no dimension measure was performed.

$$
M V(\%)=\frac{\left(M_{t}-M_{0}\right)}{M_{0}} \times 100
$$

Where, $\mathrm{MV}$ is the mass variation (\%), $\mathrm{M}_{0}$ is the initial mass (g) and $\mathrm{M}_{\mathrm{t}}(\mathrm{g})$ is the mass of the sample after the absorption.

\subsection{Study of CHX absorption and release using $U V$-vis}

UV-Vis technique was applied to evaluate the absorption and release of CHX. For it, a CHX standard curve was obtained at $232 \mathrm{~nm}$. This wave numbers were selected based on the standard curve of $\mathrm{CHX}$ at different concentrations. During the second step of $\mathrm{S}_{\text {PHB-Chi-CHX }}$ production, the residue of CHX in the supernatant was determined by UV-VIS and the amount of CHX absorbed was determined by the difference between the main solutions in comparison with the residue. After the lyophilisation, the samples were immersed in $14 \mathrm{~mL}$ of physiological solution $(\mathrm{pH} 4)$ to evaluate the $\mathrm{CHX}$ release profile. The study lasted for a total of 10 days and for each time point an aliquot was withdrawn $(4 \mathrm{~mL})$ of sample for analysis. The addition of buffer solutions to the tube alters the drug concentration and Equation 2 was used to correct it ${ }^{22}$.

$$
F C=\left(\frac{V_{0}}{V_{0}-V_{\text {aliq }}}\right)^{n-1}
$$

Where, FC (dimensionless) is the correction factor, $\mathrm{n}$ is the number of times the aliquots were taken, $\mathrm{V}_{0}(\mathrm{~mL})$ is equal to the initial volume and $\mathrm{V}_{\text {aliq }}(\mathrm{mL})$ is the aliquot volume. 


\subsection{Scanning electron microscopy}

For cross-section analysis, the matrices were fractured in liquid nitrogen and coated with a thin layer of sputtered gold. The gold-coated samples were then observed in a scanning electron microscope (JEOL, JSM-6460 LV) operating at 10 $\mathrm{kV}$ or (VEGA3 TESCAN) operating at $20 \mathrm{kV}$.

\subsection{Antibacterial activity of PHB/Chi matrices loaded with CHX by disk-agar diffusion method}

Before the microbiological evaluation, $\mathrm{S}_{\text {PHB-Chi-CHX }}$ samples were sterilized with ethylene oxide, to avoid any contamination. The antibacterial activity of $\mathrm{S}_{\text {PHB-Chi-CHX }}$ matrices and its drug delivering efficiency was assessed by adapted disk-agar diffusion test (Kirby- Bauer method). The traditional disk sample was substituted by $\mathrm{S}_{\text {PHB-Chi-CHX }}$ matrices. S. aureus, P. aeruginosa and E. coli were used in the analysis, since they are among the most common pathogens that cause infection in several body sites.

The Petri dishes containing Soy-casein agar were inoculated with $P$. aeruginosa or $S$. aureus or $E$. coli. After 2 days incubating, $\mathrm{S}_{\text {PHB-Chi-CHX }}$ matrices were placed on the surface of inoculated plates and were incubated at $36,5^{\circ} \mathrm{C}$ for $24 \mathrm{~h}$. In the sequence, the inhibition halo around the samples was defined and measured as the diameter of the inhibition zone excluding the samples.

\section{Results and Discussion}

$\mathrm{S}_{\text {PHB-Chi }}$ matrices were successfully produced by compressing molding. The resulting samples were immersed in acidic solution to evaluate the mass variation according to Equation 1 and the results may be observed in Figure 1, and, when the mass variation is positive, swelling of the sample occurs. However, negative variations are associated with mass loss due to leaching of Chi. This drastic condition was adopted precisely to evaluate the barrier effect of PHB, which has a hydrophobic characteristic and, under the adopted conditions, acidified medium with acetic acid, it is not soluble.

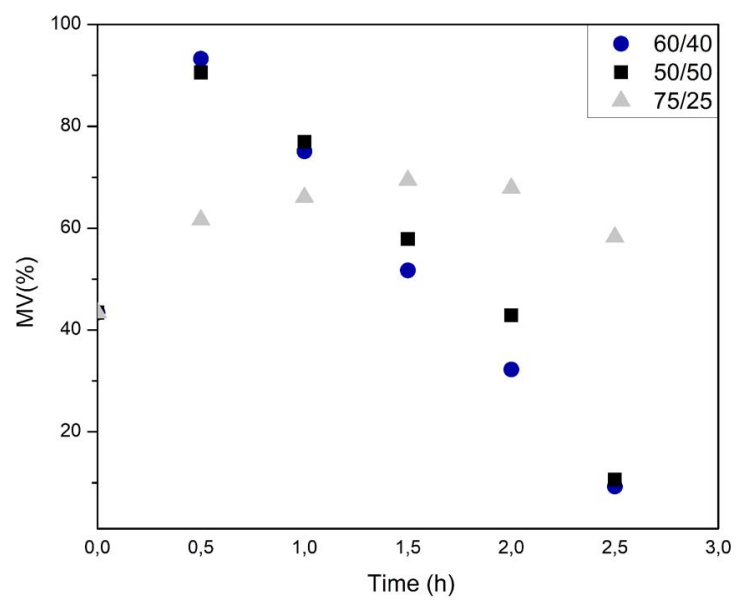

Figure 1. Mass Variation Data in Acidic medium.
The $60 / 40$ and $50 / 50$ samples, in the first 30 minutes of analysis, obtained a positive MV between 80 and $90 \%$, which can be considered as high values of MV. This occurs, probably, because of the cationic charges. When the amine groups in the Chi molecules are ionized to ammonium ion in acidic aqueous media, there are cationic repulsive forces between polymer molecules ${ }^{23}$. The content of acetic acid within the matrix is associated with diffusive processes, which are time dependent, so there must be a sufficient amount of acid for the solubilization to occur. This fact justifies the initial mass gain followed by the loss of mass due to the solubility of Chi.

However, after this period, they began to lose mass, dissolving, which confirms that the $\mathrm{pH}$ of the medium directly affects the solubility of $\mathrm{Chi}^{24}$, and, consequently, alters the mass of the matrices. The $25 / 75$ sample presented a MV increasing up to $1,5 \mathrm{~h}$ of analysis, presenting in this point the maximum of $46 \%$ and, then, like the others, also began to lose mass, but, in the meantime, the degradation was lower. The increase of the PHB content in the samples induced a higher barrier effect, making the samples with the highest percentage of Chi to be dissolved more easily. The Chi capability to swell into PHB matrices can be used to generate 3D scaffolds using a freeze-drying process. Since the concentration and $\mathrm{pH}$ have an effect on the mass variation of the matrices, the application of this matrix to the scaffold production, drug incorporation and release was evaluated.

The samples were loaded with CHX and the amount of CHX absorbed as function of both, time and samples composition, were monitored by UV-Vis (Figure 2). According to Figure 2, the Chi amount affects the CHX solution absorption. The sample with $50 \%$ of Chi trends to absorb more CHX than the others. It is possible to imply that PHB acts as a barrier to the absorption process of the CHX solution in the same way that it acted as a barrier to the diffusion of acetic acid. Indeed, the matrices have amphiphilic behavior, where the hydrophobic and hydrophilic parts are respectively associated with PHB and Chi. Moreover, these results can be related to the Chi particles distribution on the matrices.

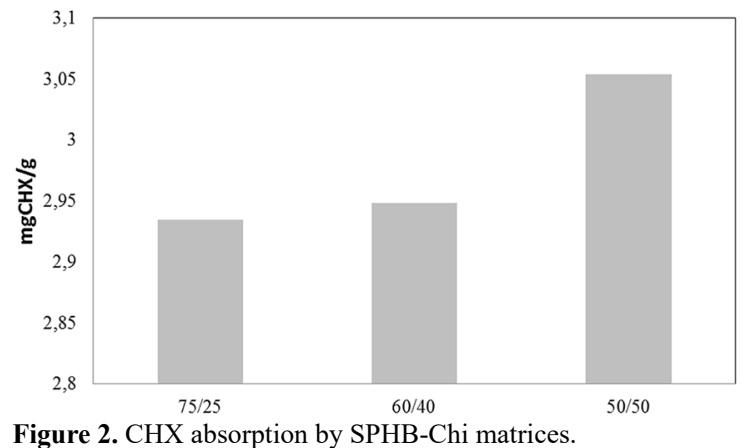

In this work, CHX release by all samples was evaluated by UV-Vis and the obtained data are shown in Figure 3. 


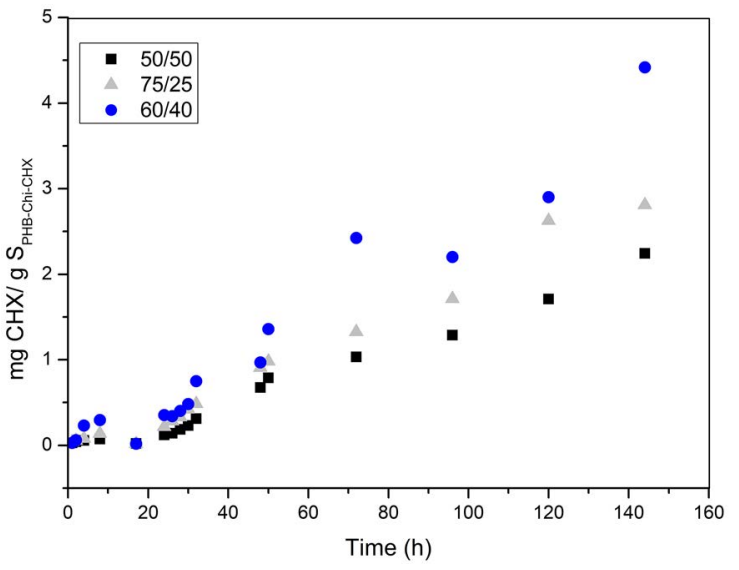

Figure 3. CHX release by $\mathrm{S}_{\text {PHB-Chi-CHX }}$ matrices.

The CHX release profile differs of some that are observed in the literature for devices based on $\mathrm{Chi}^{6,18,19}$. This occurs due to the fact that, probably, the matrices properties, such as Chi pores distribution, may imply discontinuity in the materials and, consequently, in the drug release behavior. In Figure 3, as indicated, it is possible to observe three defined behaviors, as function of time for all compositions studied. The first change in the release behavior occurs after ten hours.

Higuchi and Korsmeyer-Peppas models are widely used to evaluate the delivery behaviour of medical devices ${ }^{3}$. However, $\mathrm{S}_{\text {PHB-Chi-CHX }}$ matrices do not attend to the basic requirements of these models. Among them, the following:

i. Swelling and dissolution of the polymer matrix should be negligible;

ii. The diffusion of the drug must be constant;

iii. The film must be perfectly dipped in the middle (environment).

Due to the fact presented above the mathematical Power Law model was applied to describe CHX release. It was used the mass variation data obtained from Equation 1. The release data for all compositions studied were well adjusted by Equation 3 and Equation 4 (y, $\left.\mathrm{mg}_{[\mathrm{CHX}]} / \mathrm{g}_{[\text {SPHB-Chi-CHX] }}\right), \mathrm{R}^{2}>0.95$.

$$
\begin{aligned}
& y=A\left|t-t_{c}\right|^{b}, \text { if } t<t_{c} \\
& y=A\left|t-t_{c}\right|^{c}, \text { if } t<t_{c}
\end{aligned}
$$

Where, $\mathrm{t}$ is the immersion time (related to the release time in the medium, $\mathrm{h})$ and $\mathrm{A}\left(\mathrm{mg}_{[\mathrm{CHX}]} \mathrm{h}^{-\mathrm{b}} / \mathrm{g}_{\text {[SPHB-Chi-CHX] }}\right.$ or $\left.\mathrm{mg}_{[\mathrm{CHX}]} \mathrm{h}^{-\mathrm{c}} / \mathrm{g}_{[\text {[SPHB-Chi-CHX] }}\right), \mathrm{b}$ (dimensionless), c (dimensionless) and $\mathrm{t}_{\mathrm{c}}$ (critical time, $\mathrm{h}$ ) are constants related to the characteristics of the matrices, such as composition and morphology. The obtained values of the parameters $A, B$ and $t_{c}$ are shown in Table 1. The model indicates that for $\mathrm{t}<\mathrm{t}_{\mathrm{c}}$, a resistance to CHX delivery occurs, while for $\mathrm{t}>\mathrm{t}, \mathrm{CHX}$ delivery is improved (c $>$ b for all studied compositions). It also suggests a data interruption that can be related to change in morphology.

SEM analyses were performed in order to investigate changes in the matrices morphology throughout the drug release study (Figure 4). It was observed that the morphology of the samples changed over time. It can explain changes in CHX delivery behavior observed in Figure 3. These results indicate that the matrices properties also may be altered by the medium, since the $\mathrm{pH}$ of $\mathrm{CHX}$ solution varies between 6 and $7^{25}$. Analyzing the SEM images, it is possible to observe that the matrices thickness increases as function of time. The swelling indicates the increase of medium into the sample due the diffusion process, as observed in the analysis performed in acid medium. However, it was possible due to the presence of Chi in the samples and these results are in agreement with the literature ${ }^{20,23,26}$.

The UV spectra and their respective derivatives were used to, qualitatively, assess the loss of Chi during the release study of CHX, since it is possible to achieve the separation of overlapping peaks by differentiation of a zero-order spectrum and obtaining consecutive derivative spectra ${ }^{27}$. Hence, the spectrum of CHX was compared to the spectrum of the medium where the CHX release occurred (Figure 5). This result is in agreement with the SEM analysis that showed the formation of pores as a function of the studied release time of $\mathrm{CHX}$. This fact is interesting because the matrices produced are capable of absorbing and releasing $\mathrm{CHX}$ and, in addition, during the liberation process, the Chi exit occurs, allowing the formation of a scaffold (three-dimensional porous structure) of PHB.

The potential of $\mathrm{S}_{\text {PHB-Chi-CHX }}$ matrices of releasing CHX against microorganisms was evaluated by the disk-diffusion method. According to the inhibition halo, it was possible to determine if the released $\mathrm{CHX}$ was enough to control the growth of different microorganisms. It was possible to observe an inhibition halo, for all matrices, when they were deposited in Petri dishes and inoculated with the three groups of microorganisms. The results are confirming the potential of CHX to inhibit $S$. aureus, $P$. aeruginosa and E. coli. Additionally, it can be concluded that $\mathrm{CHX}$ was released efficiently by the matrices with different $\mathrm{PHB} / \mathrm{Chi}$ proportions. Apparently, there is no significant difference in the halo size between the $\mathrm{PHB} / \mathrm{Chi}$ groups, as observed in Figure 6.

\begin{tabular}{|c|c|c|c|}
\hline \multirow{2}{*}{ Parameters } & \multicolumn{3}{|c|}{ Samples } \\
\hline & $75 / 25$ & $60 / 40$ & $50 / 50$ \\
\hline$A\left(\mathrm{mg}_{[\mathrm{CHX}]} \mathrm{h}^{-\mathrm{b}} / \mathrm{g}_{[\text {SPHB-Chi-CHX] }}\right.$ or $\left.\mathrm{mg}_{[\text {[CHX] }} \mathrm{h}^{-\mathrm{c}} / \mathrm{g}_{[\text {SPHB-Chi-CHX] }}\right)$ & 0.0477 & 0.0413 & 0.0450 \\
\hline$B$ (dimensionless) & 0.3195 & 0.0173 & 0.1202 \\
\hline$C$ (dimensionless) & 0.9138 & 0.8183 & 0.8582 \\
\hline$t_{c}($ dimensionless $)$ & 15.782 & 20.552 & 16.990 \\
\hline
\end{tabular}

Table 1. Parameters values of Equations 3 and 4. 


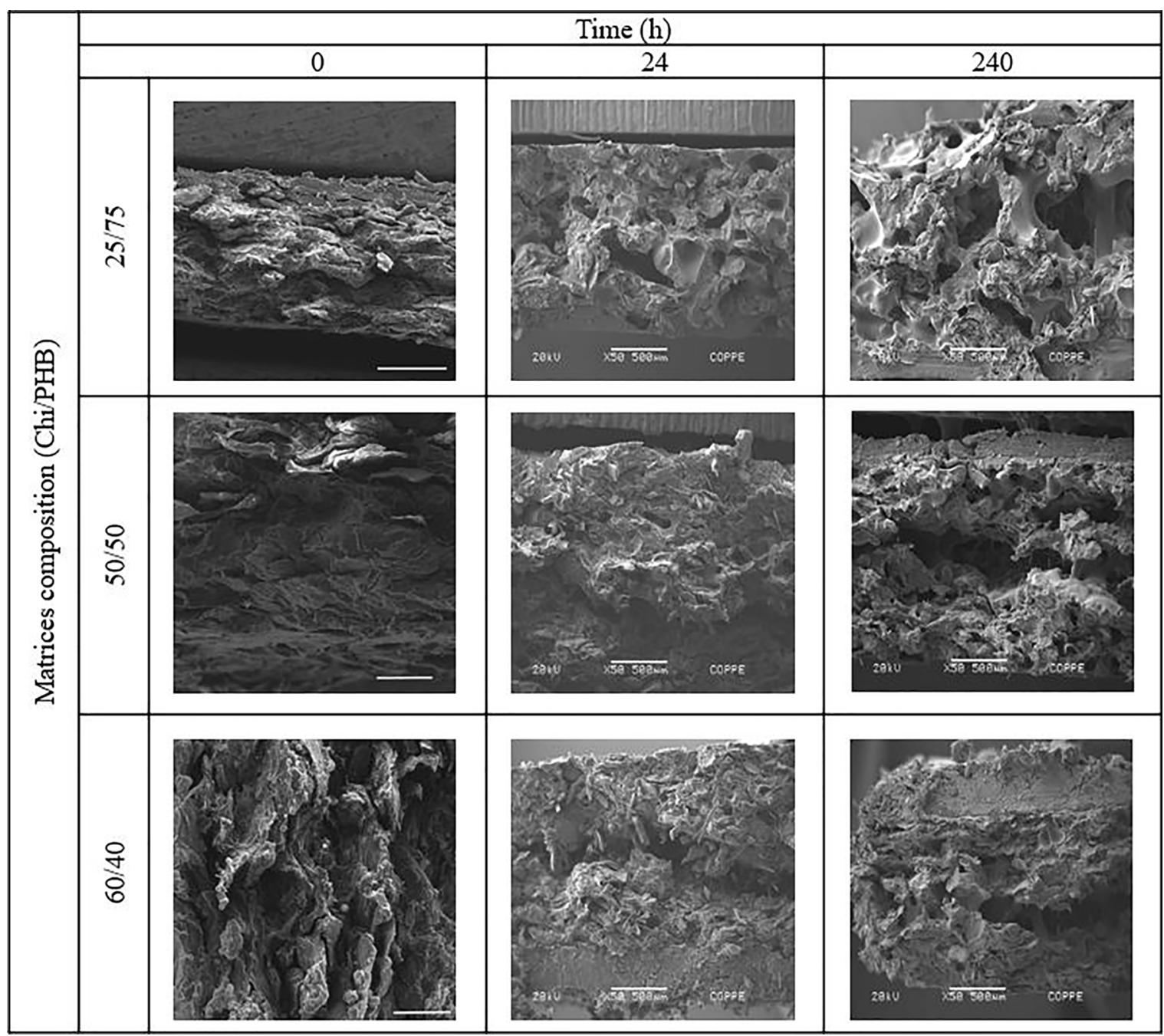

Figure 4. SEM images of matrices (0) before swelling, (24) after 24 hours in physiological solution to CHX delivery and (240) after 10 days in physiological solution to CHX delivery.
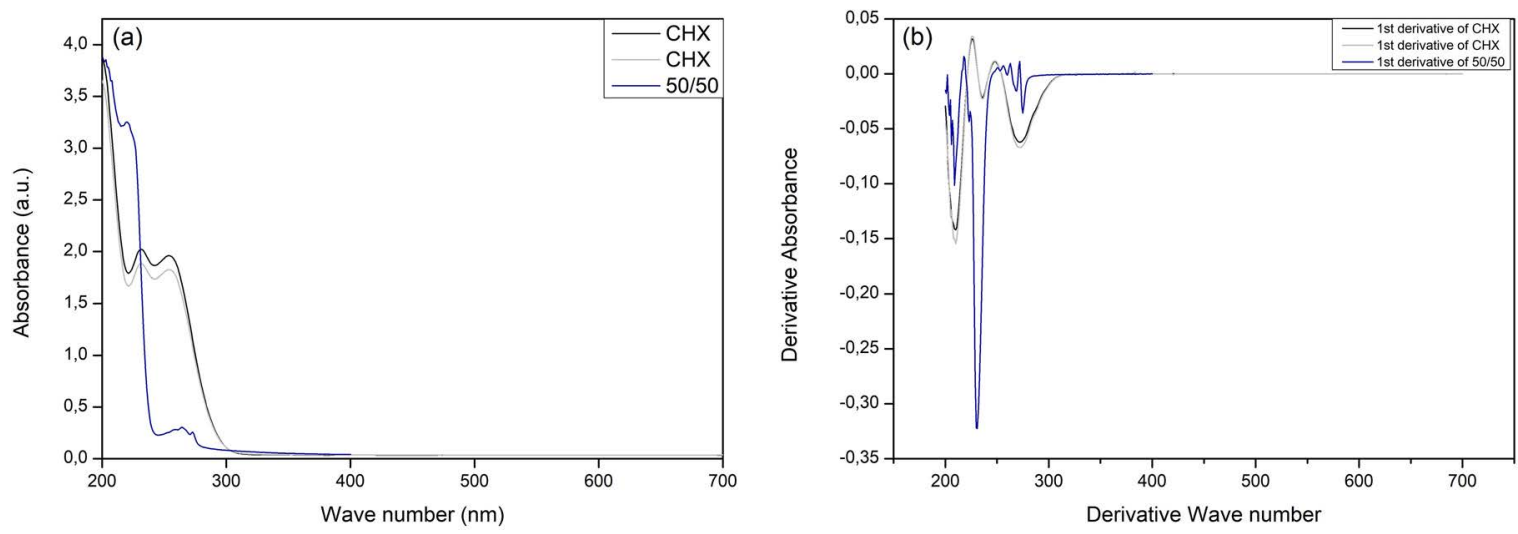

Figure 5. (a) UV spectra of CHX and release medium of sample 50/50 and (b) respective derivatives. 

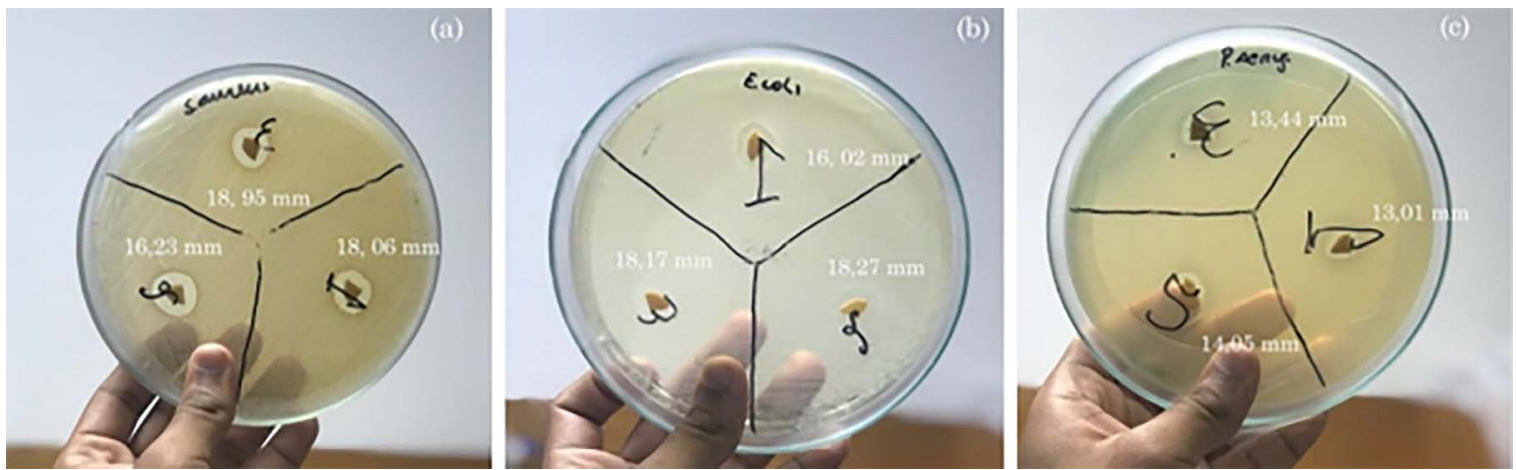

Figure 6. Petri dishes with inhibition halos, after incubation for 24h. (a) MRSA; (b) E. coli and (c) P. aeruginosa.

\section{Conclusion}

Matrices of PHB and Chi obtained by compression molding technique were successfully loaded with $\mathrm{CHX}$. However, it was observed that the Chi amount affected the $\mathrm{CHX}$ absorption. That is, the increase in the Chi content improved the CHX absorption. This occurs due to the barrier effect related to PHB and to the fact that the matrices are amphiphilic. The CHX delivery study was performed in physiological solution. It was observed that the matrices morphology was altered during the studied period and a porous structure was obtained, and, the porous formation is important due to the capability of forming 3D scaffolds. The CHX release was modelled by the power law model and its coefficients were affected by composition. Moreover, for the same sample, the delivery profile changes as function of time and, consequently, changes in the model parameters occur. The potential of $\mathrm{PHB} / \mathrm{Chi}$ matrices against microorganisms was evaluated by the diskdiffusion method. The results confirmed the potential of CHX to inhibit $S$. aureus, $P$. aeruginosa and E. coli. Accordingly, this system seems to be a potential candidate to oral tissue engineering applications, with dual function: at first, local release of $\mathrm{CHX}$; subsequently, the remaining structure will work as a scaffold, guiding the tissue growth.

\section{Acknowledgments}

This study was financed in part by the Coordenação de Aperfeiçoamento de Pessoal de Nível Superior - Brasil (CAPES) - Finance Code 001. The authors would also like to acknowledge CNPq and FAPERJ for their financial support.

\section{References}

1. Xie J, Li A, Li J. Advances in pH-Sensitive Polymers for Smart Insulin Delivery. Macromolecular Rapid Communications. 2017;38(23):1700413.

2. Pramanik P, Halder D, Jana SS, Ghosh S. pH-Triggered Sustained Drug Delivery from a Polymer Micelle having the $\beta$-Thiopropionate Linkage. Macromolecular Rapid Communications. 2016;37(18):1499-1506.
3. Tiwari G, Tiwari R, Sriwastawa B, Bhati L, Pandey S, Pandey $\mathrm{P}$, et al. Drug delivery systems: An update review. International Journal of Pharmaceutical Investigation. 2012;2(1):2-11.

4. Jøraholmen MW, Vanić Z, Tho I, Škalko-Basnet N. Chitosan-coated liposomes for topical vaginal therapy: assuring localized drug effect. International Journal of Pharmaceutics. 2014;472(1-2):94-101.

5. Zhang Z, Tsai PC, Ramezanli T, Michniak-Kohnand BB. Polymeric nanoparticles-based topical delivery systems for the treatment of dermatological diseases. Wiley Interdisciplinary Reviews: Nanomedicine and Nanobiotechnology. 2013;5(3):205-218.

6. Onnainty R, Onida B, Páez P, Longhi M, Barresi A, Granero G. Targeted chitosan-based bionanocomposites for controlled oralmucosal delivery of chlorhexidine. International Journal of Pharmaceutics. 2016;509(1-2):408-418.

7. Busilacchi A, Gigante A, Mattioli-Belmonte M, Manzotti S, Muzzarelli RA. Chitosan stabilizes platelet growth factors and modulates stem cell differentiation toward tissue regeneration. Carbohydrate Polymers. 2013;98(1):665-676.

8. Cao W, Wang A, Jing D, Gong Y, Zhao N, Zhang X. Novel biodegradable films and scaffolds of chitosan blended with poly(3-hydroxybutyrate). Journal of Biomaterial Science, Polymer Edition. 2005;16(11):1379-1394.

9. Medvecky L. Microstructure and Properties of PolyhydroxybutyrateChitosan-Nanohydroxyapatite Composite Scaffolds. The Scientific World Journal. 2012;2012:537973.

10. Medvecky L, Giretova M, Stulajterova R. Properties and in vitro characterization of polyhydroxybutyrate-chitosan scaffolds prepared by modified precipitation method. Journal of Materials Science: Materials in Medicine. 2014;25(3):777-789.

11. Silva GF, da Silva TG, Gobbi VG, Portela TL, Teixeira BN, Mendonça TS, et al. Swelling degree prediction of polyhydroxybutyrate/ chitosan matrices loaded with "Arnica-do-Brasil". Journal of Applied Polymer Science. 2019;136(32):47838.

12. Pujana MA, Pérez-Álvarez L, Iturbe LCC, Katime I. Water dispersible $\mathrm{pH}$-responsive chitosan nanogels modified with biocompatible crosslinking-agents. Polymer. 2012;53(15):31073116 .

13. Mendonça RH, Meiga TD, da Costa MF, Thiré RMSM. Production of 3D scaffolds applied to tissue engineering using chitosan swelling as a porogenic agent. Journal of Applied Polymer Science. 2013;129(2):614-625. 
14. Iordanskii AL, Rogovina SZ, Kosenko RY, Ivantsova EL, Prut EV. Development of a biodegradable polyhydroxybutyratechitosan-rifampicin composition for controlled transport of biologically active compounds. Doklady Physical Chemistry. 2010;431(2):60-62.

15. Kim WH, Lee SB, Oh KT, Moon SK, Kim KM, Kim KN. The release behavior of $\mathrm{CHX}$ from polymer-coated titanium surfaces. Surface and Interface Analysis. 2008;40(3-4):202-204.

16. Ambrogi V, Pietrella D, Nocchetti M, Casagrande S, Moretti V, De Marco S, et al. Montmorillonite-chitosan-chlorhexidine composite films with antibiofilm activity and improved cytotoxicity for wound dressing. Journal of Colloid and Interface Science. 2017;491:265-272.

17. Hosseini S, Kassaee M, Elahi SH, Bolhari B. A novel binary chlorhexidine-chitosan irrigant with high permeability, and long lasting synergic antibacterial effect. Nanochemistry Research. 2018;3(1):92-98.

18. Sandri G, Rossi S, Bonferoni MC, Ferrari F, Mori M, Caramella C. The role of chitosan as a mucoadhesive agent in mucosal drug delivery. Journal of Drug Delivery Science and Technology. 2012;22(4):275-284.

19. Senel S, Ikinci G, Kaş S, Yousefi-Rad A, Sargon MF, Hincal AA. Chitosan films and hydrogels of chlorhexidine gluconate for oral mucosal delivery. International Journal of Pharmaceutics. 2000;193(2):197-203.

20. Giunchedi P, Juliano C, Gavini E, Cossu M, Sorrenti M. Formulation and in vivo evaluation of chlorhexidine buccal tablets prepared using drug-loaded chitosan microspheres. European Journal of Pharmaceutics and Biopharmaceutics. 2002;53(2):233-239.
21. Berwig E. Preparação e caracterização de filmes de poliuretano com potencial aplicação na liberação controlada de fármacos. [Dissertation]. Florianópolis: Federal University of Santa Catarina, School of Physical and Mathematical Sciences; 2006.

22. Shifrovitch Y, Binderman I, Bahar H, Berdicevsky I, Zilberman M. Metronidazole-loaded bioabsorbable films as local antibacterial treatment of infected periodontal pockets. Journal of Periodontology. 2009;80(2):330-337.

23. Park H, Park K, Kim D. Preparation and swelling behavior of chitosan-based superporous hydrogels for gastric retention application. Journal of Biomedical Materials Research Part A. 2006;76(1):144-150.

24. Sargin I, Arslan G. Chitosan/sporopollenin microcapsules: Preparation, characterization and application in heavy metal removal. International Journal of Biological Macromolecules. 2015; 75:230-238.

25. Bonesvoll P, Lökken P, Rölla G. Influence of concentration, time, temperature and $\mathrm{pH}$ on the retention of chlorhexidine in the human oral cavity after mouth rinses. Archives of Oral Biology. 1974;19(11):1025-1029.

26. Viswanathan K, Monisha P, Srinivasan M, Swathi D, Raman M, Raj GD. Chlorhexidine-calcium phosphate nanoparticles - Polymer mixer based wound healing cream and their applications. Materials Science and Engineering. C. 2016;67:516-521.

27. Kuś S, Marczenko Z, Obarski N. Derivative UV-VIS Spectrophotometry in Analytical Chemistry. Chemia Analityczna (Warsaw). 1996;41:899-927. 